\title{
Concentrative ER export of the serotonin transporter relies exclusively on an interaction with Sec24C
}

\author{
Sonja Sucic ${ }^{1}$, Ali El-Kasaby ${ }^{1}$, Subhodeep Sarker ${ }^{1}$, Harald H Sitte ${ }^{1}$, Philippe Marin², Michael Freissmuth ${ }^{1 *}$ \\ From 16th Scientific Symposium of the Austrian Pharmacological Society (APHAR) \\ Vienna, Austria. 25-27 November 2010
}

\section{Background}

The transporters for serotonin (SERT), dopamine (DAT) and noradrenaline (NET) form the monoamine neurotransmitter transporter family. They are primarily responsible for the termination of neurotransmission via rapid reuptake of neurotransmitters from the synaptic cleft. We have previously shown that the C-terminus of SERT plays a key role in trafficking and folding of the transporter. Mutations in this region of the protein (specifically at sites $\mathrm{PG}^{601-602}$ and $\mathrm{RI}^{607-608}$ ) cause intracellular retention of SERT, hence abolishing substrate uptake and reducing inhibitor binding. In the current study, we explored isoform-specific interaction of COPII component Sec24 proteins with monoamine transporters, to study the mechanistic nature of their ER export.

\section{Methods}

Our initial studies involving mass spectrometry revealed that SERT directly interacts with Sec24C. To confirm these data, we subsequently used the siRNA approach to individually knock down the four mammalian Sec24 isoforms A, B, C or D in immortalised cervical cancer cells (HeLa). Fourty-eight hours subsequent to siRNA transfections, the cells were transfected with YFP-tagged transporter plasmids and substrate uptake assays were performed after an additional $24 \mathrm{~h}$.

\section{Results}

While gene silencing of Sec24A, B or D led to no changes in SERT function, that of Sec24C alone dramatically impaired serotonin uptake. It is therefore evident that SERT requires specifically Sec $24 \mathrm{C}$ for its export from the ER and reaching its site of action at the cell membrane. Our further data verify residues $\mathrm{RI}^{607-608}$ as the ER export motif on SERT C-terminus, which mediates the interaction with Sec24C and in turn the formation of COPII vesicles. Surprisingly, the related transporters, DAT and NET, require Sec24D for their ER export, which is consistent with reports in the literature regarding other NSS transporters (e.g. GAT-1 and GLYT).

\section{Conclusions}

ER export and trafficking of SERT occurs in a unique manner, judged by its exclusive interaction with Sec24C, and is different to other NSS transporters.

\section{Author details}

${ }^{1}$ Institute of Pharmacology, Center for Physiology and Pharmacology, Medical University of Vienna, 1090 Vienna, Austria. ${ }^{2}$ Institut de Génomique Fonctionnelle, CNRS UMR 5203, INSERM U661, Universités Montpellier I \& II, 34094 Montpellier Cedex 5, France.

Published: 16 November 2010

doi:10.1186/1471-2210-10-S1-A11

Cite this article as: Sucic et al.: Concentrative ER export of the serotonin transporter relies exclusively on an interaction with Sec24C. BMC Pharmacology 2010 10(Suppl 1):A11.

\footnotetext{
* Correspondence: michael.freissmuth@meduniwien.ac.at

'Institute of Pharmacology, Center for Physiology and Pharmacology,

Medical University of Vienna, 1090 Vienna, Austria

Full list of author information is available at the end of the article
} 